\title{
ESTRUTURA FITOSSOCIOLÓGICA DE UM TRECHO DE VEGETAÇÃO ARBÓREA NO PARQUE ESTADUAL DO RIO DOCE - MINAS GERAIS, BRASIL ${ }^{1}$
}

\author{
Waldomiro de Paula Lopes ${ }^{2}$ \\ Alexandre Francisco da Silva ${ }^{3}$ \\ Agostinho Lopes de Souza ${ }^{4}$ \\ João Augusto Alves Meira Neto ${ }^{3}$
}

Recebido em 01/11/2001. Aceito em 08/05/2002.

\begin{abstract}
RESUMO - (Estrutura fitossociológica de um trecho de vegetação arbórea no Parque Estadual do Rio Doce - Minas Gerais, Brasil). O presente trabalho teve como objetivo estudar a estrutura de um estande de Floresta Estacional Semidecidual Submontana no Parque Estadual do Rio Doce (19 $29^{\prime}-19^{\circ} 48^{\prime} \mathrm{S}$ e $\left.42^{\circ} 28^{\prime}-42^{\circ} 38^{\prime} \mathrm{W}\right)$. Os indivíduos de porte arbóreo foram amostrados pelo método de quadrantes. Adotaram-se dois procedimentos distintos, dos quais um com inclusão dos indivíduos mortos e em outro não. Foram alocados 200 pontos amostrais, dispostos em 20 linhas paralelas, ao longo de uma encosta. Foram encontradas 143 espécies, pertencentes a 109 gêneros reconhecidos e 38 famílias botânicas. A análise fitossociológica demonstrou predominância das espécies Bixa arborea, Guatteria schomburgkiana, Joannesia princeps, Aparisthmium cordatum, Pseudopiptadenia contorta e Carpotroche brasiliensis. $\mathrm{O}$ tamanho das populações e sua distribuição pelo ambiente foram os fatores decisivos para o destaque apresentado por essas espécies, com exceção de $P$. contorta, que esteve representada por poucos indivíduos, contudo de grandes dimensões. As distribuições diamétricas e de alturas evidenciaram poucos indivíduos de grande porte, fato que aliado ao histórico do local, que registra um incêndio em 1967, e à baixa ocorrência de espécies associadas a ambientes climácicos, sugere que o trecho de floresta estudado encontra-se em estádio médio de sucessão secundária.
\end{abstract}

Palavras-chave - Fitossociologia, estrutura fitossociológica, floresta estacional

\begin{abstract}
Phytosociological structure of a stand of arboreal vegetation in Rio Doce State Park - Minas Gerais, Brazil). The objective of the present work was to study the structure of a stand of Submontane Semi-deciduous Forest, in Rio Doce State Park (19 $29^{\prime}-19^{\circ} 48^{\prime}$ S e $\left.42^{\circ} 28^{\prime}-42^{\circ} 38^{\prime} \mathrm{W}\right)$. In order to sample the tree component, the point-centered-quarter method was employed according to two different protocols: in one including the dead individuals, and in the other excluding them. A total of 200 sampling points were allocated in 20 parallel lines, along a hillside. A total of 143 species, belonging to 109 recognized genera and 38 botanical families was found. The main species were Bixa arborea, Guatteria schomburgkiana, Joannesia princeps, Aparisthmium cordatum, Pseudopiptadenia contorta and Carpotroche brasiliensis. The size of the populations and their distribution throughout the environment determined the prominence of these species, with the exception of P. contorta, which was represented by few large individuals. The diameter and height distributions evidenced few individuals of great size, a fact probably linked to a history of burning a 1967. The fire history and the low occurrence of climax species suggest that the forest stand studied is in a medium stage of secondary succession.
\end{abstract}

Key words - Phytosociology, phytosociological structure, semideciduous forest

\footnotetext{
${ }^{1}$ Parte da Dissertação de Mestrado do primeiro autor; apoio: CAPES e FAPEMIG.

${ }^{2}$ Mestre em Botânica - Universidade Federal de Viçosa, wplopes@ hotmail.com.

${ }^{3}$ Universidade Federal de Viçosa, Departamento de Biologia Vegetal, 36.571-000, Viçosa, Minas Gerais, Brasil.

${ }^{4}$ Universidade Federal de Viçosa, Departamento de Engenharia Florestal, 36.571-000, Viçosa, Minas Gerais, Brasil.
} 


\section{Introdução}

O Parque Estadual do Rio Doce (PERD) constitui-se na maior área remanescente de floresta tropical no estado de Minas Gerais, com aproximadamente $36.000 \mathrm{ha}$. Sua vegetação faz parte da Floresta Estacional Semidecidual Submontana (Veloso et al. 1991).

Na década de 60 o PERD teve grande parte de sua vegetação destruída por incêndios, tendo o maior deles ocorrido em 1967. Após esse período não houve registro de focos de incêndio de maiores dimensões, ficando a vegetação sob processo de regeneração natural. Com isso, as florestas atualmente encontradas são em grande parte secundárias, intercaladas com trechos que ainda possuem características primárias. Considerando-se o modelo e o nível de intervenção humana na região, que resultou na destruição de extensas áreas da cobertura vegetal original, o PERD assume uma posição de destaque na preservação dos recursos naturais regionais.

No tocante à vegetação, apesar de destacarse no cenário das Unidades de Conservação do Estado, o PERD ainda carece de estudos mais detalhados. Apenas trabalhos iniciais de reconhecimento da vegetação ou de táxons específicos foram realizados até o momento. Dentre esses destacam-se SOCT \& CETEC (1981), Gilhuis (1986), Pedralli et al. (1986), Graçano et al. (1998), Scudeller \& CarvalhoOkano (1998), Bortoluzzi (2000), Gonçalves (2000) e Temponi (2001).

Objetivou-se, com este trabalho, determinar a estrutura fitossociológica da vegetação arbórea de um trecho de floresta no Parque Estadual do Rio Doce.

\section{Material e métodos}

Área de estudo - os limites do PERD (19 29'$19^{\circ} 48^{\prime} \mathrm{S}$ e $42^{\circ} 28^{\prime}-42^{\circ} 38^{\prime} \mathrm{W}$ ) incluem-se em três municípios no leste do Estado (Marliéria, Dionísio e Timóteo), na região conhecida como "Vale do Aço". Sua criação data de 1944, sendo administrado pelo Instituto Estadual de florestas desde 1962 (IEF 1994).

O relevo apresenta-se predominantemente com contornos suaves, na forma de colinas, planícies e vales planos sedimentares, com altitude variando de 230 a 515 metros. Cerca de $21 \%$ da área do Parque corresponde à classe de relevo plano, ondulado a montanhoso (39\%) e fortemente ondulado a montanhoso (34\%) (CETEC 1982). Segundo CETEC (1978), no PERD os solos são predominantemente argilosos e álicos, com acidez média a elevada, predominando aqueles com horizonte $\mathrm{B}$ textural. Apresenta ainda um complexo hídrico de aproximadamente 50 lagos, que ocupam quase $6 \%$ de sua área (Feio et al. 1998).

O clima da região é do tipo Aw (clima tropical úmido de savana, megatérmico), segundo a classificação de Köppen, com um período de chuvas e outro de estiagem bem definidos. As precipitações atingem até $235 \mathrm{~mm}$ no mês de dezembro, reduzindo a 9mm em agosto (CETEC 1978; Gilhuis 1986). Os valores médios anuais de precipitação, umidade relativa do ar e temperatura, calculados a partir de registros dos últimos vinte anos, estão em torno de $1.300 \mathrm{~mm}$, $79 \%$ e $23^{\circ} \mathrm{C}$, respectivamente.

A escolha do local de desenvolvimento do presente trabalho baseou-se, principalmente, na fisionomia que se desejava estudar. Optou-se por trabalhar nas proximidades da trilha do Vinhático, em um trecho de floresta em bom estado de conservação, em regeneração desde um incêndio ocorrido no final da década de 60 . Os trabalhos de campo foram realizados durante os anos de 1996 e 1997.

Amostragem fitossociológica - para o estudo fitossociológico, utilizou-se o método de quadrantes (Cottam \& Curtis 1956), com o cálculo da distância corrigida individual modificada por Martins (1991), sendo 
amostrados todos os indivíduos arbóreos que apresentavam circunferência do tronco, a $1,30 \mathrm{~m}$ do solo (CAP), maior ou igual a $15 \mathrm{~cm}$. A orientação dos quadrantes em cada ponto amostral foi aleatória, conforme proposto por Mueller-Dombois \& Ellenberg (1974). O distanciamento entre os pontos foi determinado empregando-se a fórmula da distância mínima (Martins 1991), obtendo-se um valor final de 12m. Foram fixadas dez linhas amostrais, com 20 pontos em cada uma, também eqüidistantes em $12 \mathrm{~m}$, alocadas perpendicularmente à declividade do terreno. Amostrou-se o trecho de floresta cujo histórico e homogeneidade fisionômica indicavam o menor nível de perturbação.

Durante a coleta de dados foram realizadas duas amostragens distintas, incluindo em uma delas apenas os indivíduos vivos e na outra os vivos e os mortos, desde que ainda em pé. Os dados coletados de cada indivíduo morto foram a distância até o ponto e a CAP. O procedimento consistiu do seguinte: uma vez encontrado um indivíduo morto tomavam-se suas medidas e amostrava-se o próximo indivíduo vivo, no mesmo quadrante.

De cada indivíduo vivo foram medidas a CAP, a altura total (utilizando-se de um telêmetro) e coletadas amostras de material botânico. Todo material fértil obtido encontra-se depositado no Herbário VIC, da Universidade Federal de Viçosa. Duplicatas desses espécimens também serão incluídas ao acervo do Herbário do PERD.

Utilizando-se o programa Fitopac 1 (Shepperd 1994), calculou-se o Índice de Diversidade de Shannon (Brower \& Zar 1984) e a Equabilidade (Pielou 1975), bem como s seguintes parâmetros de abundância (Mueller-Dombois \& Ellenberg 1974): Densidade Absoluta (DA), Densidade Relativa (DR), Dominância Absoluta (DoA), Dominância Relativa (DoR), Freqüência Absoluta (FA), Freqüência Relativa (FR), Valor de Cobertura (VC) e Valor de Importância (VI).

Para a apresentação dos táxons adotou-se o sistema de classificação proposto por Cronquist
(1981), exceto para o grupo das Leguminosas, que foi tratado como uma única família (Engler \& Diels 1936 apud Lawrence 1951). A confirmação da grafia dos nomes e sinonímia, foi obtida consultando-se o Índice de Espécies do Royal Botanic Gardens - Kew (1993), ou literatura mais recente. A grafia dos autores seguiu a abreviação sugerida por Brumit \& Powell (1992).

Distribuição diamétrica - para a avaliação da estrutura horizontal utilizou-se a distribuição de frequiência das classes de diâmetro, fixas em $5 \mathrm{~cm}$, que foram analisadas a partir da construção de histograma de frequiência (Spiegel 1977). A primeira classe incluiu os indivíduos com até $10 \mathrm{~cm}$ de diâmetro de caule a 1,30m do solo. Os dados utilizados na presente análise foram gerados utilizando-se o programa Fitopac 1 (Shepperd 1994) e Diamfito (Mota 1995).

Estrutura vertical - para esta análise foram consideradas as alturas totais dos indivíduos amostrados. Elaborou-se a distribuição de freqüência das classes de altura, sendo apresentada graficamente na forma de histograma de frequiência. Para a determinação do número mínimo de classes, bem como sua amplitude, utilizou-se a fórmula proposta por Spiegel (1977), apresentada a seguir:

$\mathrm{NC}=1+3,3 \log (\mathrm{n}) ; \mathrm{IC}=\frac{\mathrm{A}}{\mathrm{NC}}$, em que

$\mathrm{NC}=$ número de classes;

$\mathrm{n}=$ número de indivíduos;

IC = intervalo de classe; $\mathrm{e}$

$\mathrm{A}=$ amplitude de classe (altura máxima - altura mínima).

\section{Resultados e discussão}

Tomando-se a classificação proposta por Gilhuis (1986), o estande de vegetação estudado enquadrou-se na categoria denominada de Mata 
Alta. Isso pode ser afirmado considerando-se a florística relacionada pelo mesmo autor como característica desse ambiente, onde se destacavam as espécies Astrocaryum aculeatissimum e Joannesia princeps, que foram encontradas com densidade elevada na área escolhida para o presente trabalho. Além da localização acrescentam-se aos dados florísticos, sinais de carbonização, estrutura da vegetação arbórea e baixa ocorrência de epífitas, bambusóides e gramíneas. Ainda, segundo aquele autor, esse tipo de vegetação recobre aproximadamente $30 \%$ da área total do PERD.

Estrutura fitossociológica - analisando-se os resultados da amostragem caso se incluísse a classe dos mortos, ter-se-ia um total de 23 indivíduos pertencentes a esta categoria, totalizando uma área basal de $0,6 \mathrm{~m}^{2}$. A área equivalente da amostra seria de 0,55 ha e a distância média entre as plantas de 2,61m. Neste caso, a área basal da amostragem somaria $14,06 \mathrm{~m}^{2}$, sendo a estimativa por hectare de $25,78 \mathrm{~m}^{2}$ e a densidade estimada por hectare resultaria em 1466 indivíduos. Apresentando valores de 2,88 para DR, 4,22 para DoR e 2,92 para FR, o grupo de indivíduos mortos ocuparia o quarto lugar considerando-se o VC $(3,54 \%)$ e dividiria a quarta colocação em VI com Aparisthmium cordatum (3,34\%). Ressalta-se que em se adotando esse procedimento, duas espécies não seriam amostradas: Banara kuhlmanii (Flacourtiaceae) e Euplassa sp., sendo esta última a única representante da família Proteaceae. Esses dados mostram que ao se utilizar um método de amostragem independente de área, como o de quadrantes, em regiões pouco conhecidas floristicamente, seria mais adequado a não inclusão de indivíduos mortos, se abordagens acerca da dinâmica da comunidade não constarem dos objetivos do trabalho.

Comparando-se o ordenamento das espécies em relação aos parâmetros fitossociológicos, com e sem a inclusão dos indivíduos mortos, observouse que não haveriam alterações nas colocações daquelas mais importantes quanto ao VI e ao VC, ocorrendo apenas algumas trocas de posições entre as espécies de menor destaque.

Avaliando-se os dados fitossociológicos obtidos a partir da amostragem incluindo exclusivamente espécimens vivos, foram amostrados 800 indivíduos, obtendo-se distância média de $2,5 \mathrm{~m}$ e resultando em uma área amostral de 0,51 ha e $13,73 \mathrm{~m}^{2}$ de área basal. Os valores estimados por hectare foram de 1569 indivíduos e $26,94 \mathrm{~m}^{2}$, estando próximos dos encontrados em Florestas Estacionais secundárias (MeiraNeto \& Martins 2000; Silva et al. 2000).

O Índice de Diversidade de Shannon apresentou o valor de 3,98nats/indivíduo. Esse valor representa uma diversidade elevada, em se tratando de Floresta Estacional, corroborada pelo valor de 0,8 estimado para a Equabilidade, indicando que $80 \%$ da diversidade máxima teórica foi representada nesta amostragem.

$\mathrm{Na}$ Tab. 1 estão apresentadas as espécies e seus parâmetros fitossociológicos. Inicialmente nota-se que as maiores populações pertenceram a Bixa arborea (126 indivíduos), Guatteria schomburgkiana (87), Joannesia princeps (46), Aparisthmium cordatum (43) e Carpotroche brasiliensis (22), o que é evidenciado pelos valores de Densidade Absoluta e Relativa apresentados por essas espécies. Dentre as 143 espécies encontradas, 53 foram representadas por apenas um indivíduo, correspondendo a aproximadamente $37 \%$ do total. Esse valor vem satisfazer um dos objetivos da utilização do método de quadrantes no desenvolvimento do presente trabalho, que foi o de melhor representar a composição florística do estande florestal avaliado.

Foram necessárias apenas as 12 primeiras espécies em VI para ultrapassar a metade $(50,5 \%)$ de seu valor total. Essas mesmas espécies compuseram $52,1 \%$ do VC. Esses 


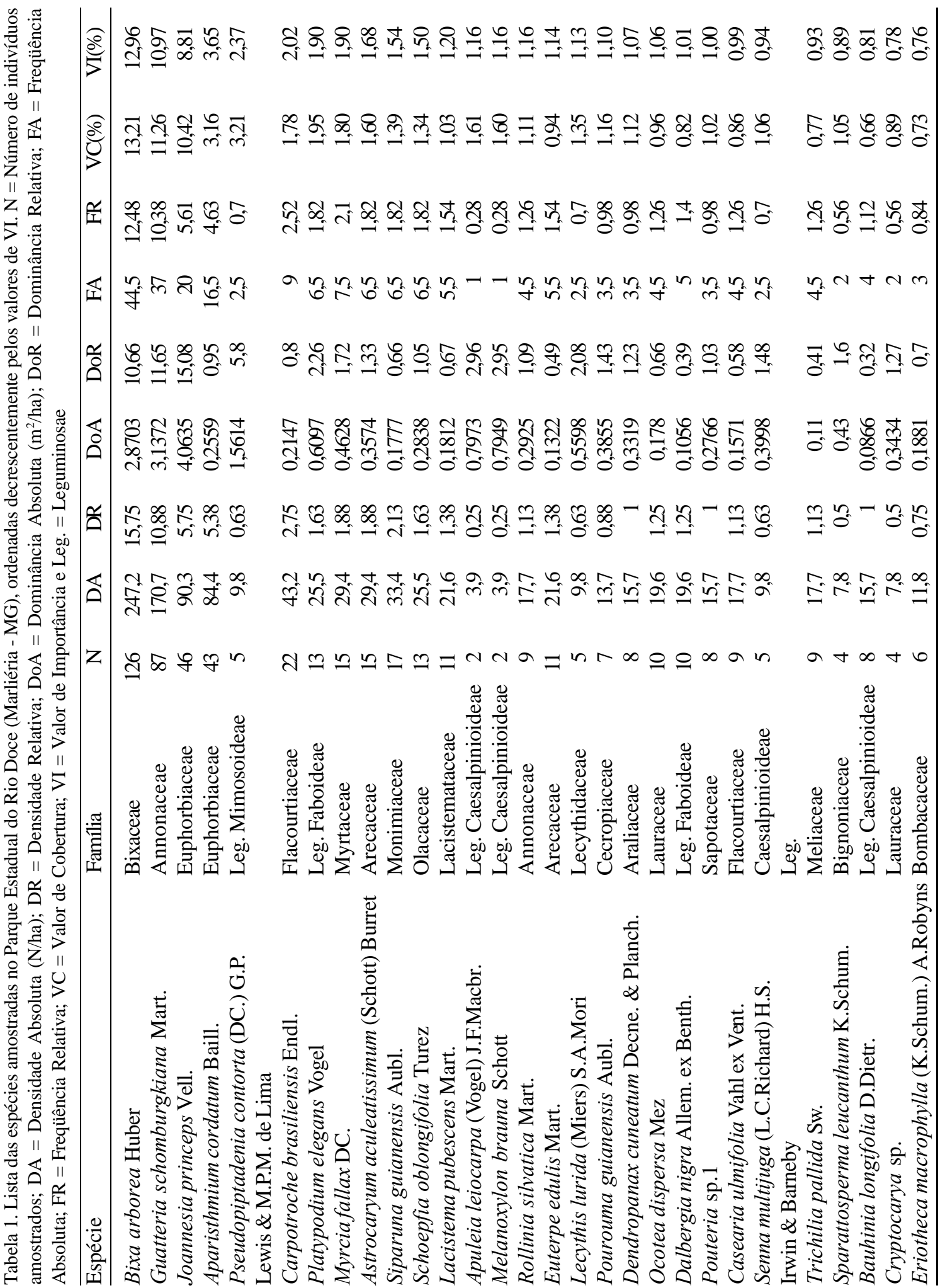




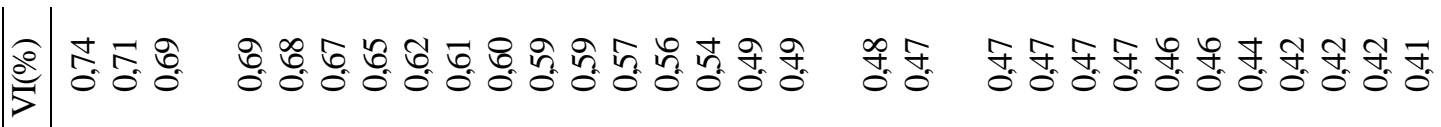

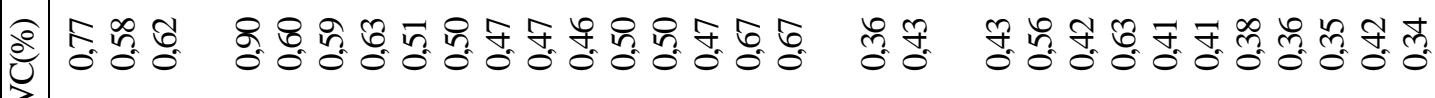

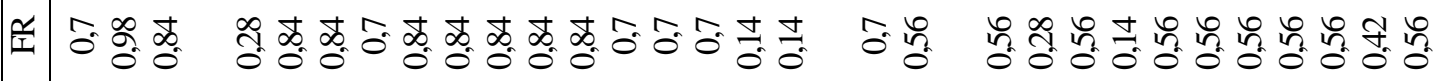

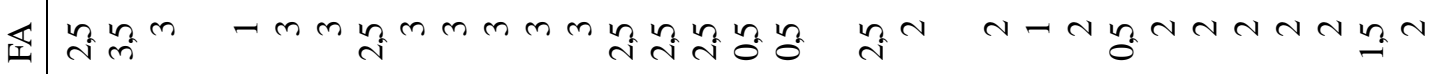

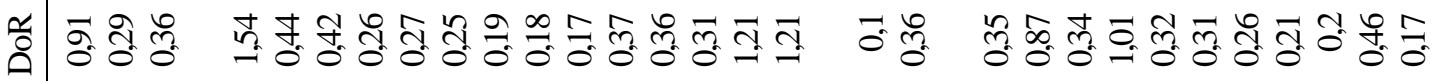

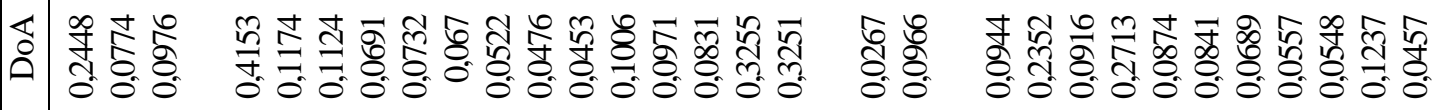

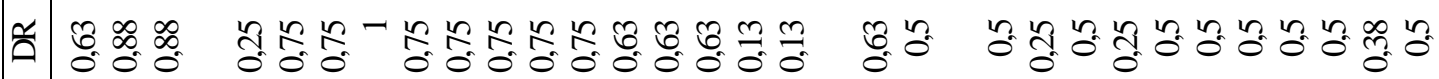

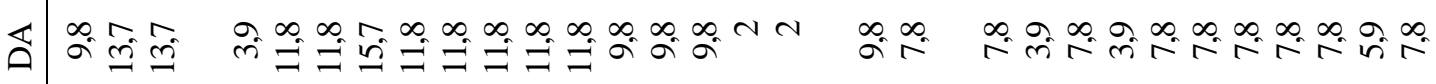
Z

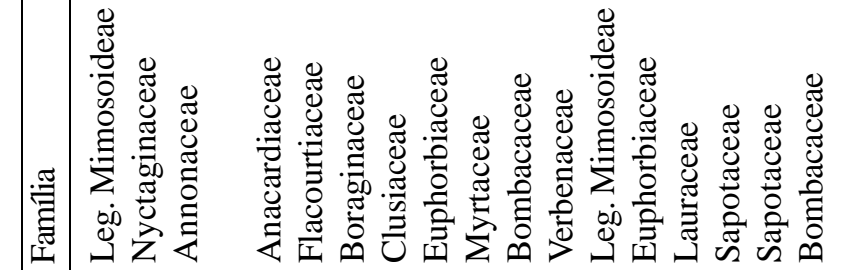

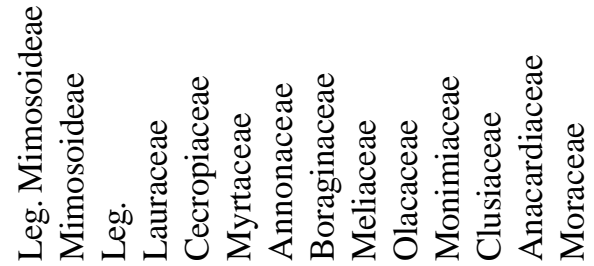

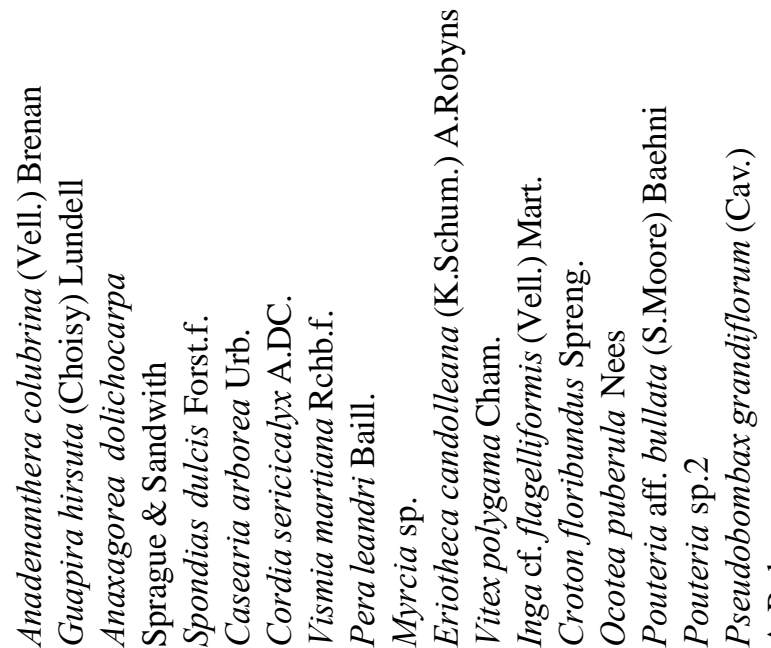

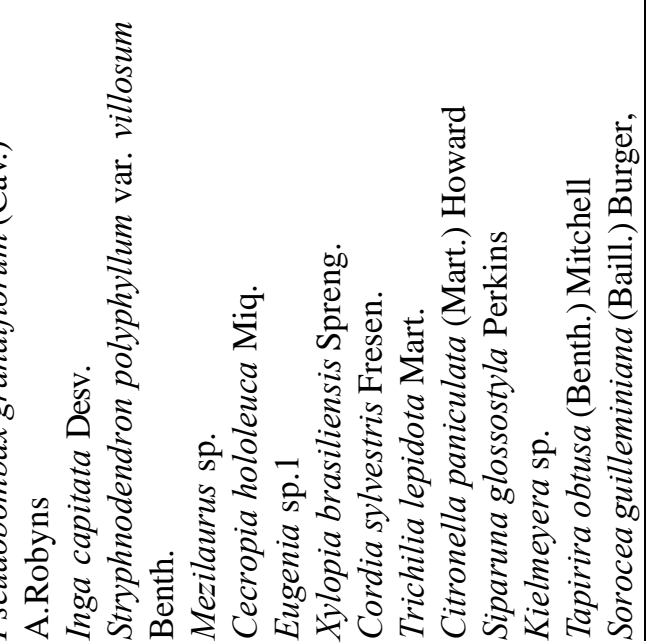




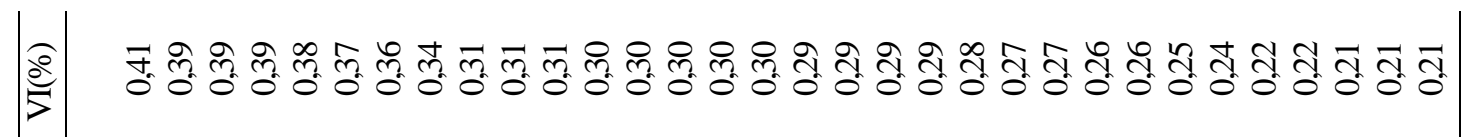

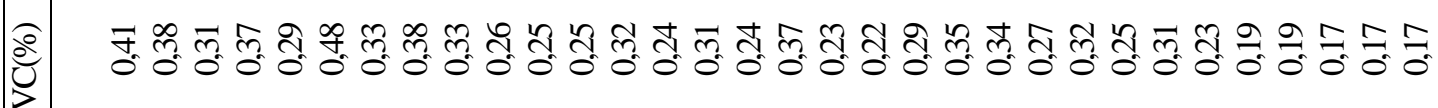

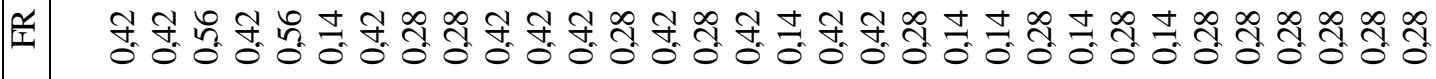

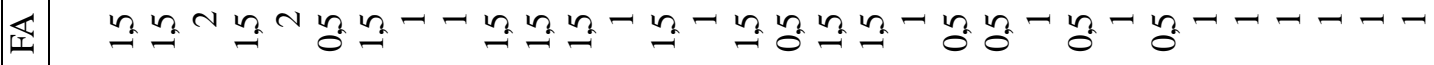

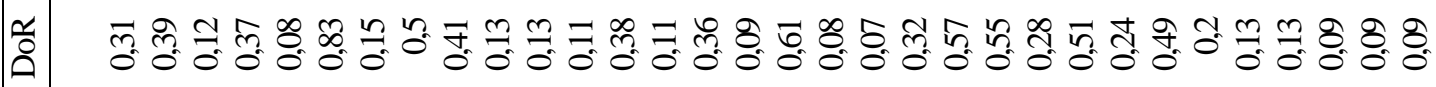

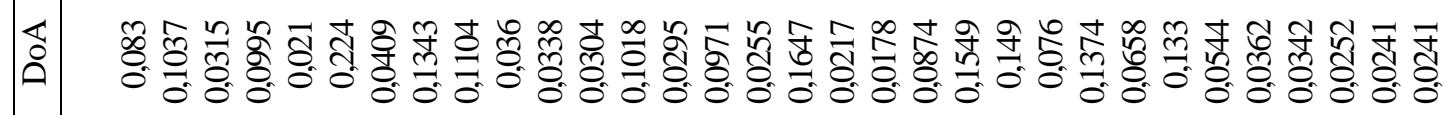

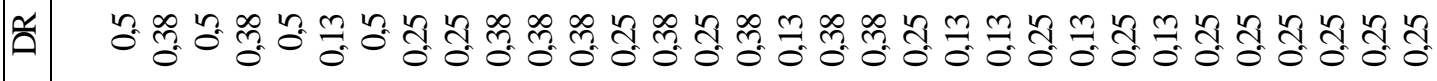

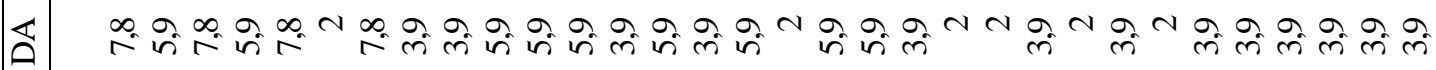

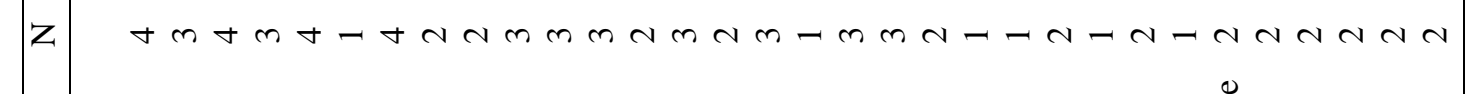

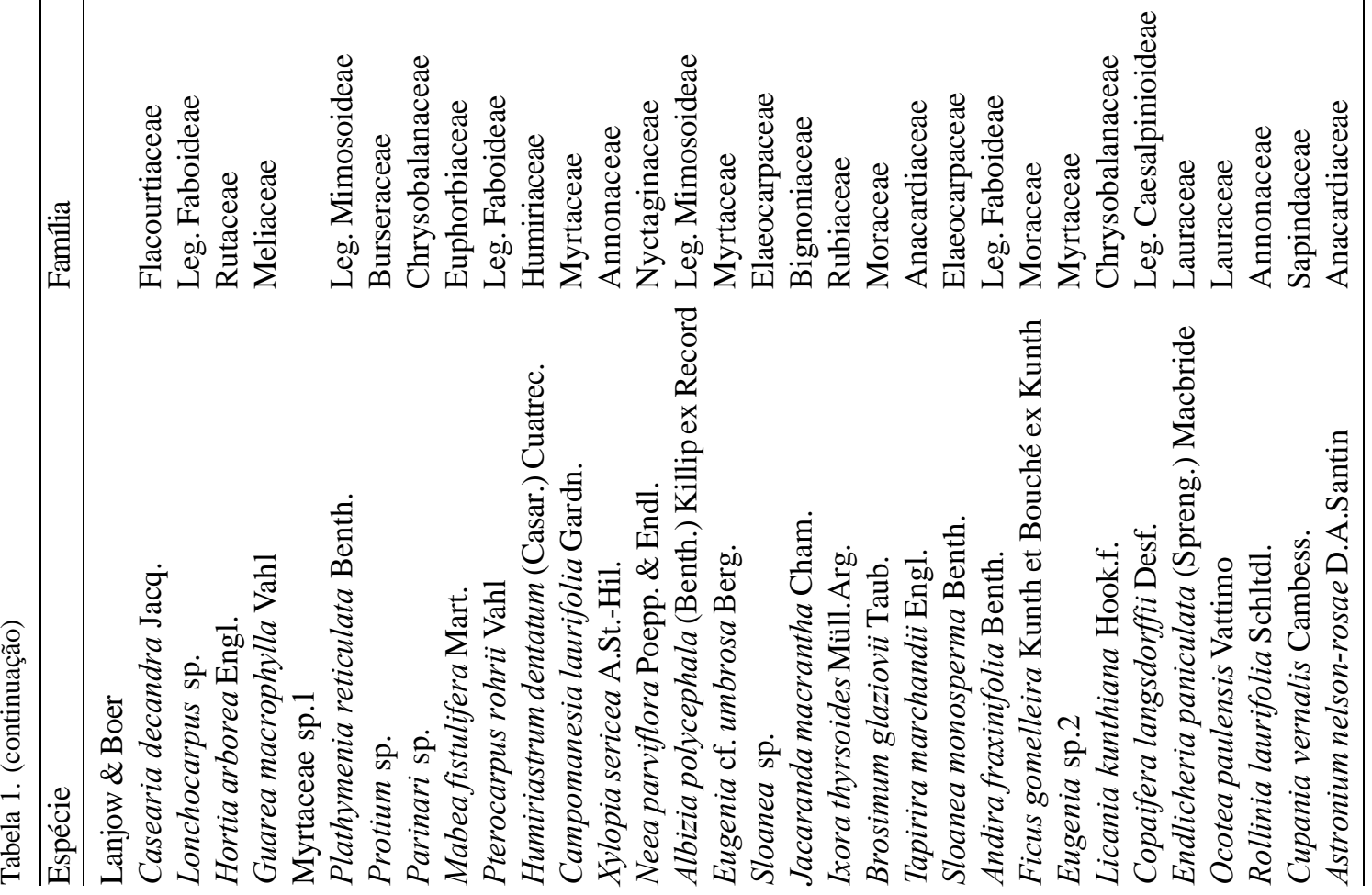




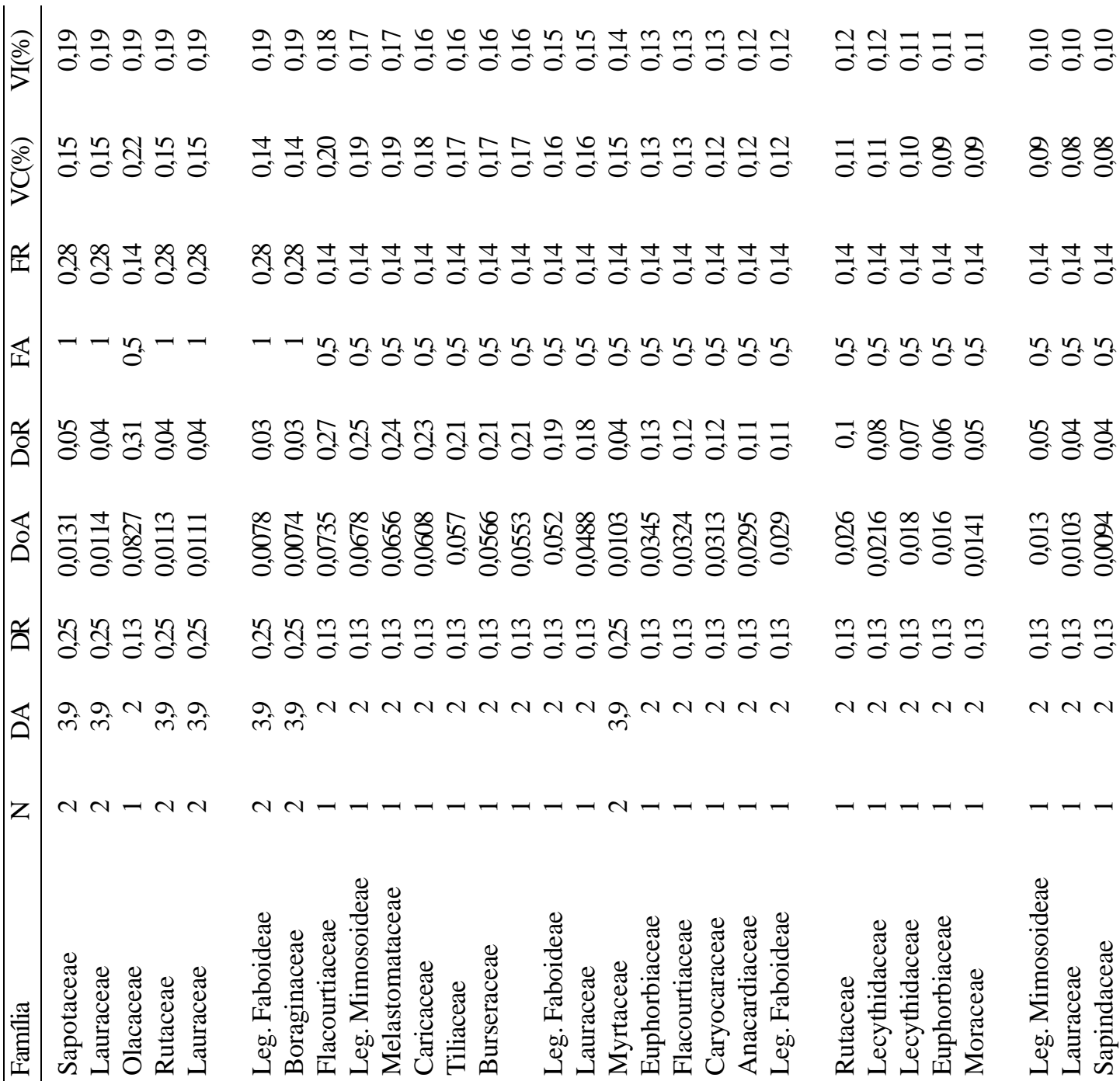

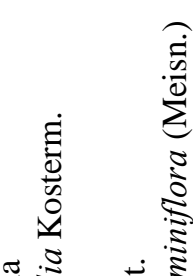

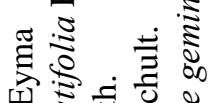

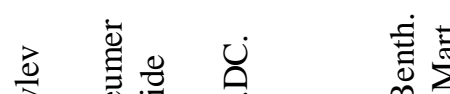

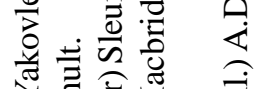

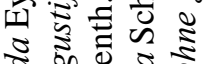
穵苛 क्षे ᄅ

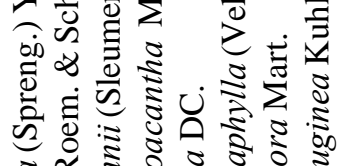

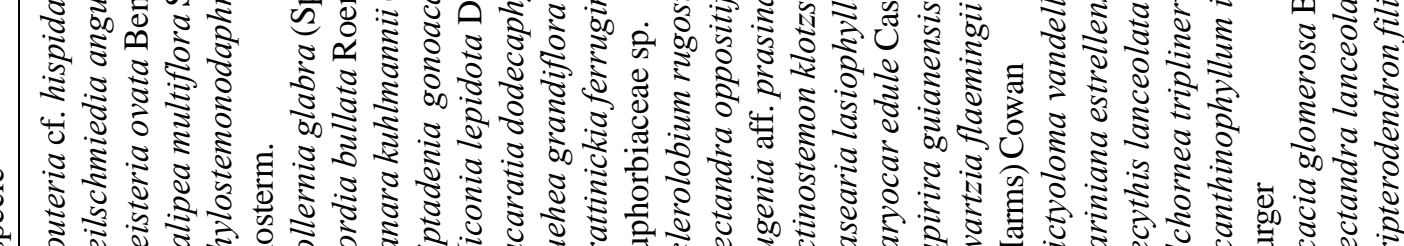

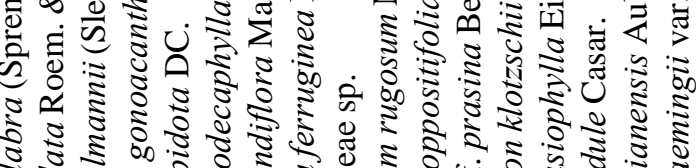

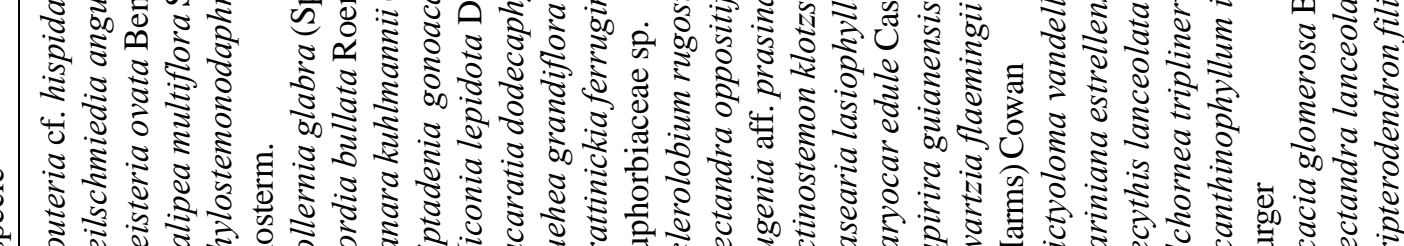

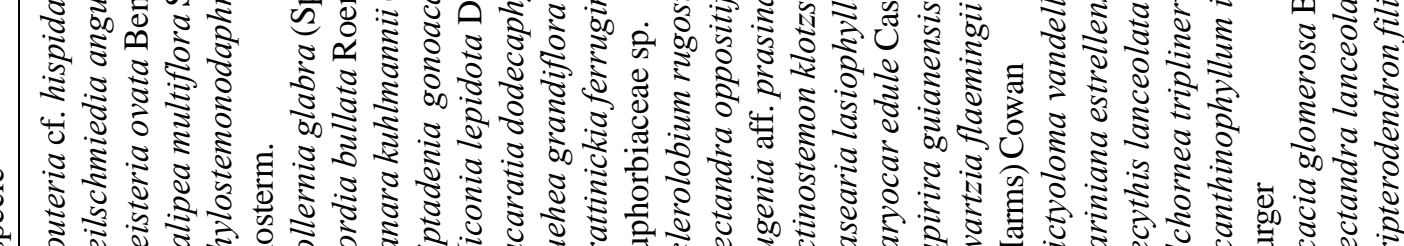

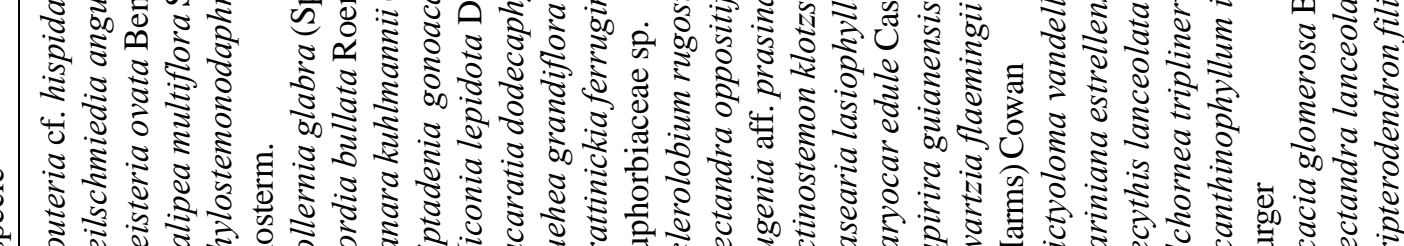

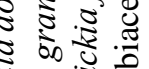

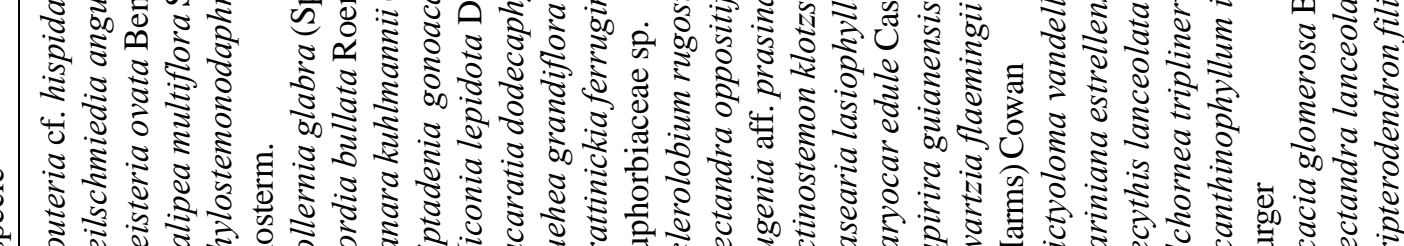
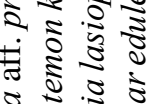

迆 势 


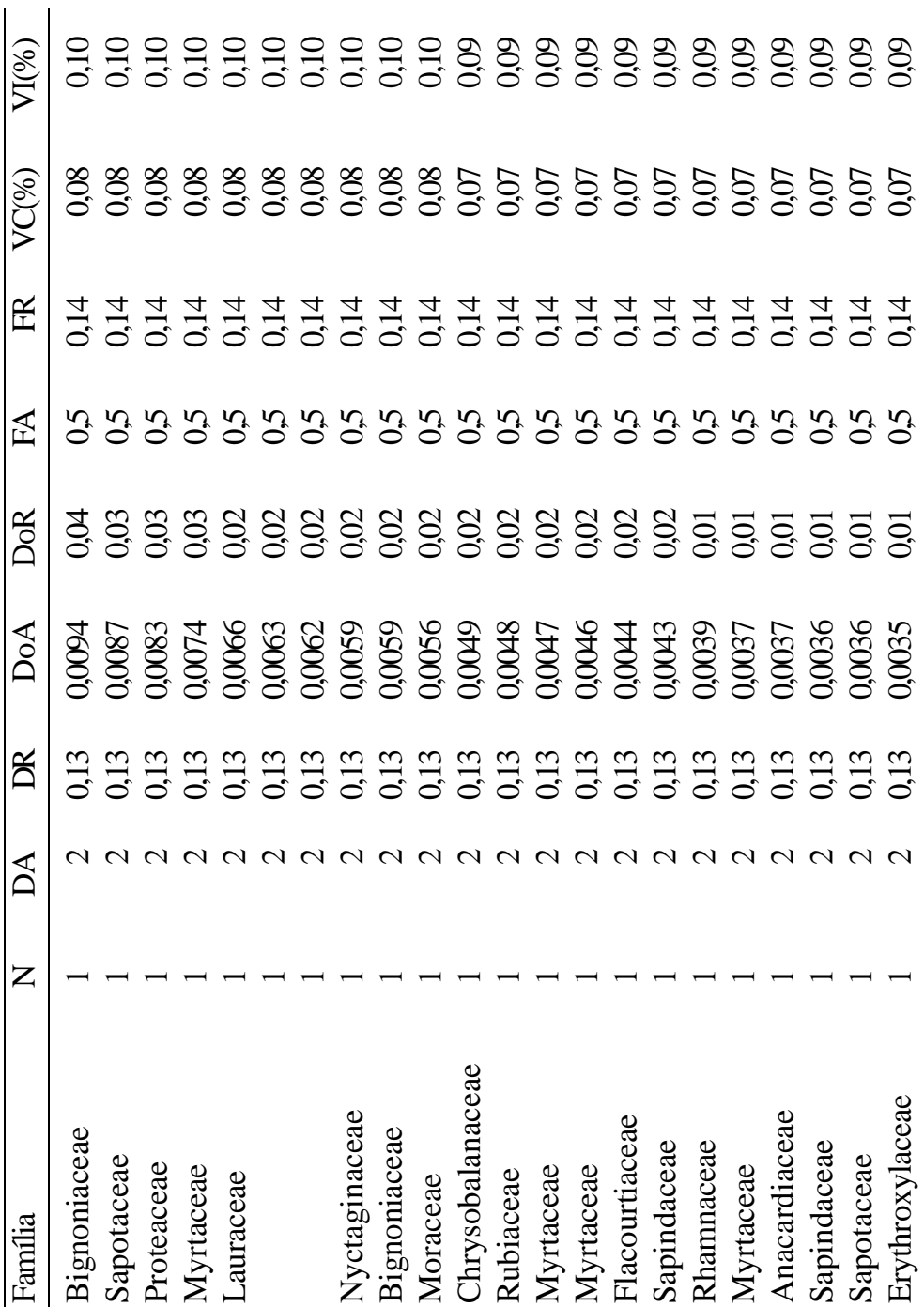


resultados demonstram que, apesar dos valores relativamente altos de Diversidade e Equabilidade encontrados, existe um número reduzido de espécies (em torno de $8 \%$ ) que apresentam dominância no ambiente, fato que é comum na maioria das florestas tropicais. Dentre essas espécies, as três primeiras contribuíram de modo mais expressivo (Bixa arborea, Guatteria schomburgkiana e Joannesia princeps), somando $32,7 \%$ do VI e $34,9 \%$ do VC. Para as duas primeiras, os parâmetros que compõem o VI participaram de maneira semelhante na sua composição. Com relação à posição ocupada por Joannesia princeps, o porte de seus representantes foi decisivo, refletindo em valores elevados de Dominância. Esse padrão também foi observado na população de Pseudopiptadenia contorta, quinta colocada em VI e quarta em VC.

O número de indivíduos e sua distribuição pelo ambiente podem ser considerados como os maiores responsáveis pela posição, na fitocenose estudada, das espécies Aparisthmium cordatum, Carpotroche brasiliensis, Siparuna guianensis e Lacistema pubescens, que por sua vez apresentaram baixos valores de Dominância. Em relação à densidade, valores elevados para esse parâmetro indicam que tais espécies apresentam-se bem adaptadas à atual sere sucessional, ou seja, são mais competitivas nas condições ambientais do momento.

$\mathrm{O}$ fato de apresentarem diferentes exigências e estratégias de ocupação, explorando distintos recursos do habitat, permite que espécies com comportamentos diversos figurem concomitantemente entre as mais importantes (VI). Esse é o caso, por exemplo, de Aparisthmium cordatum, Astrocaryum aculeatissimum, Carpotroche brasiliensis e Siparuna guianensis, que não alcançam grandes diâmetros e maiores alturas, ou Apuleia leiocarpa, Melanoxylon brauna, Platypodium elegans e Pseudopiptadenia contorta, que podem apresentar diâmetros e alturas consideráveis na maturidade.

Distribuição diamétrica - o resultado obtido a partir da distribuição de frequiência nas classes diamétricas, de todos os indivíduos amostrados (Fig. 1), é o esperado para as florestas ineqüiâneas secundárias, apresentando-se a curva na forma de um "J" invertido (Silva Júnior \& Silva 1988). A curva resultante indica que existe um decréscimo acentuado no número de indivíduos, no sentido das menores para as maiores classes diamétricas. Em função disso pode-se afirmar que a fisionomia florestal encontra-se em pleno desenvolvimento em direção a estádios mais avançados, uma vez que existe um contingente de indivíduos jovens que irão suceder àqueles que já se encontram senis ou em decrepitude.

Observando-se a Fig. 2, nota-se a ausência de representantes na penúltima classe diamétrica. A partir do gráfico elaborado, além de observações de campo, especula-se que algumas árvores sobreviveram ao incêndio ocorrido na área. Dois desses indivíduos pertencem à espécie Pseudopiptadenia contorta, estando ambos na antepenúltima classe diamétrica. A última classe foi ocupada por um representante de Melanoxylon brauna, com $71,5 \mathrm{~cm}$ de diâmetro a $1,30 \mathrm{~m}$ do solo. A inferência de que tais indivíduos sobreviveram ao fogo pode ser embasada na afirmação de Meyer et al. (1961), dentre outros autores, que comentaram o fato da distribuição de diâmetros refletir o histórico da floresta, bem como a ocorrência, no passado, de distúrbios tais como fogo, corte, doenças, ataque de insetos e outros fenômenos.

Felfili (1993) comentou que muitas inferências podem ser realizadas com relação à ecologia de cada espécie e da comunidade, a partir da análise de suas estruturas diamétricas e de alturas. A autora exemplificou o fato de ser possível avaliar, por exemplo, se as espécies estão continuamente se regenerando ou não, 


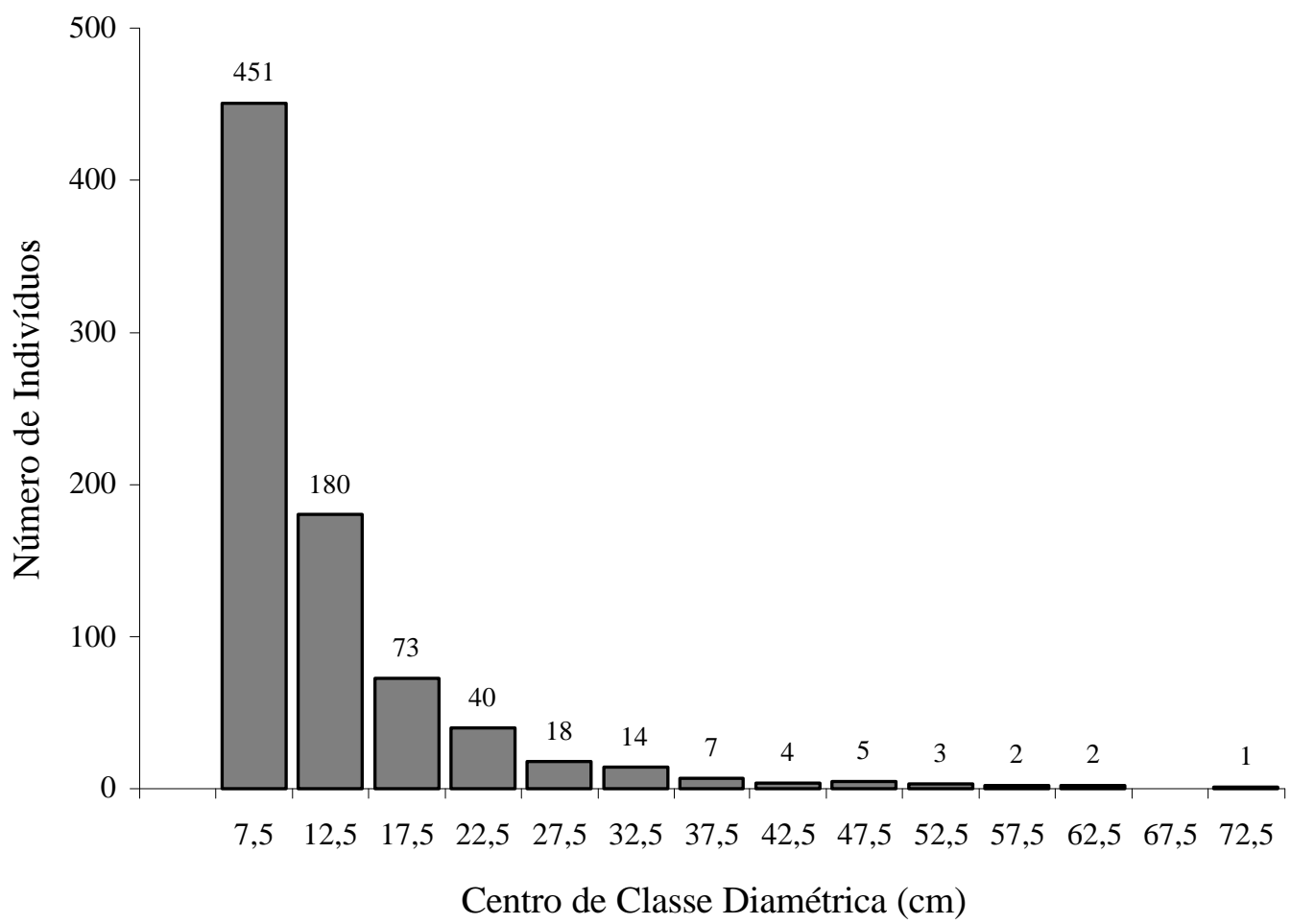

Figura 1. Distribuição de frequiência das classes de diâmetro de todos os indivíduos incluídos na amostragem fitossociológica. Parque Estadual do Rio Doce (Marliéria - MG).

ou se têm um estoque de plantas jovens suficiente para conservar, no futuro, a abundância atual. Souza \& Jesus (1994) colocaram, ainda, que a análise da distribuição diamétrica de uma espécie, ou de um grupo de espécies, informa sobre as características ecofisiológicas das mesmas.

Estrutura vertical - entre os indivíduos amostrados encontrou-se uma altura (total) máxima de 27,8m, mínima de $2,6 \mathrm{~m}$ e média de $9,4 \mathrm{~m}$. O cálculo do número de classes de altura resultou em 11 classes com amplitude de 2,5m. A distribuição dos indivíduos, em função da altura máxima alcançada por suas copas, apresentouse semelhante àquela encontrada na distribuição de diâmetros, com exceção da primeira classe, com frequiência inferior à da classe seguinte (Fig.
2). Esse comportamento parece ser comum em florestas tropicais, tendo sido observado por outros autores (Cavassan 1983; Leitão Filho 1993; Martins 1991). Uma possível explicação para tal fato estaria relacionada com o critério de inclusão de indivíduos na amostra, que provavelmente excluiu aqueles com altura correspondente à da primeira classe, mas que ainda não haviam atingido CAP de $15 \mathrm{~cm}$.

Aproximadamente $65 \%$ dos indivíduos amostrados estão concentrados entre os seis e 12 metros de altura. Apesar disso, a distribuição dos indivíduos pelas diversas classes de altura não forneceu subsídios suficientes para afirmar o número de estratos existentes, bem como determiná-los. O que se observou foi uma continuidade na distribuição das copas nas diferentes alturas. Como exemplo de espécies 


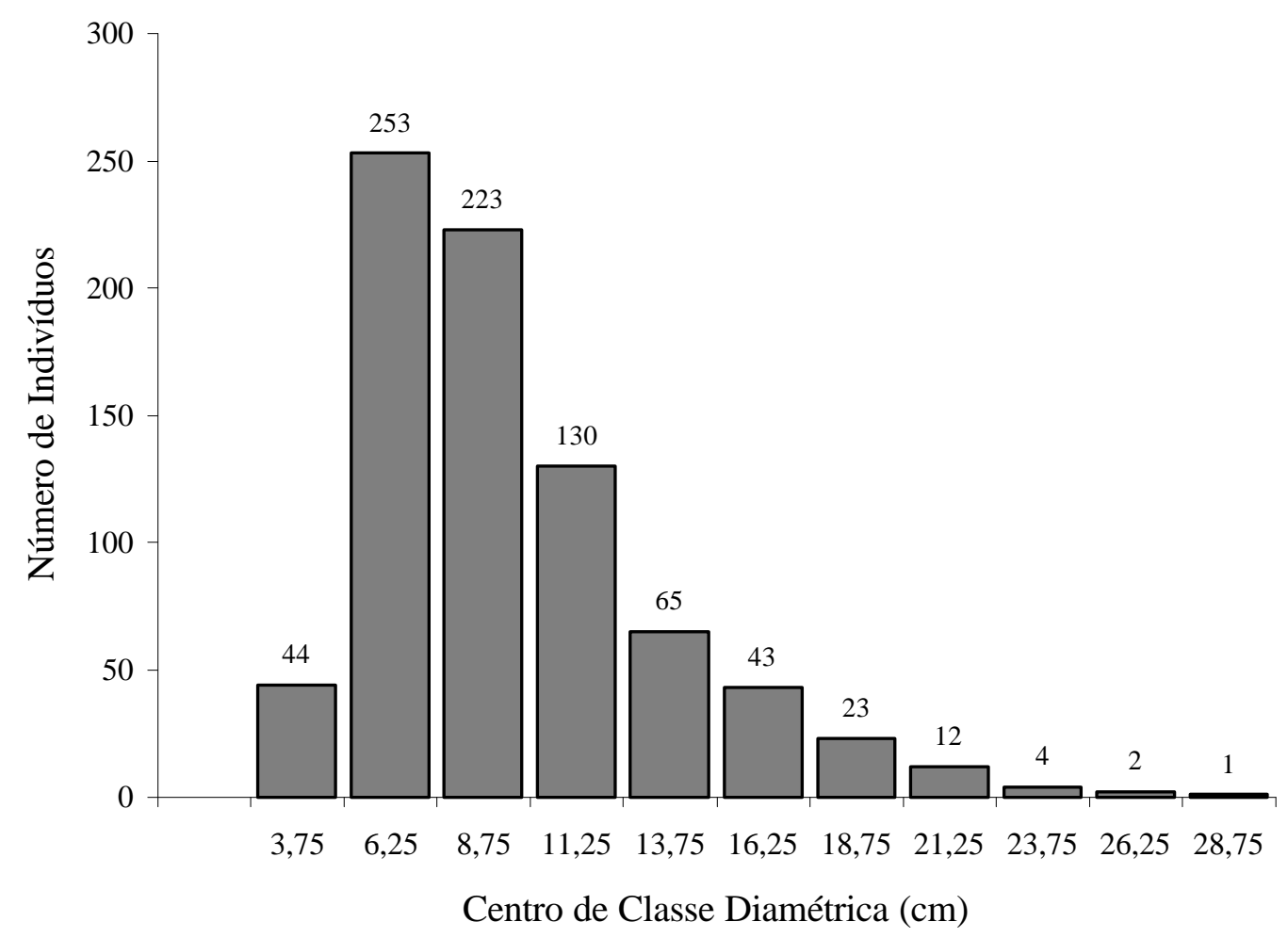

Figura 2. Distribuição de freqüência das classes de altura total de todos os indivíduos incluídos na amostragem fitossociológica. Parque Estadual do Rio Doce (Marliéria - MG).

bem representadas em todas as faixas de altura, pode-se citar Bixa arborea, Guatteria schomburgkiana e Joannesia princeps. Espécies como Anaxagorea dolichocarpa, Astrocaryum aculeatissimum e Siparuna guianensis apresentaram a maioria de seus indivíduos com alturas inferiores aos sete metros. Por fim, Pseudopiptadenia contorta e Apuleia leiocarpa foram registradas apenas nas faixas superiores. Melo \& Mantovani (1994) estudando o componente arbóreo de uma comunidade vegetal na ilha do Cardoso (SP), também observaram que, para a comunidade como um todo, ocorreu uma distribuição contínua dos indivíduos nas diversas classes de altura. Contudo, outros autores obtiveram resultados que permitiram a visualização de uma concentração de copas em determinados estratos, os quais foram delimitados (Silva \& Leitão Filho 1982; Pagano et al. 1987; Peixoto et al. 1995).

A partir das análises realizadas, observações de campo e recorrendo-se ao histórico de perturbações da floresta estudada, acredita-se que o estande avaliado encontra-se em estádio médio de sucessão secundária. Essa suposição também se fundamenta na baixa ocorrência de espécies reconhecidas como de ambiente climácico, como por exemplo Acanthinophyllum ilicifolium, Myrciaria floribunda, Rhamnidium elaeocarpum e Urbanodendron verrucosum, relacionadas com um indivíduo cada uma, e Anaxagorea dolichocarpa, amostrada com sete indivíduos. Essa constatação, remontandose à ocorrência de um incêndio nessa área no final da década de 60, demonstra que a recomposição daquele ambiente está avançando 
continuadamente. Isso pode ser explicado observando-se que, após o fogo, alguns indivíduos de grande porte, em plena maturidade fisiológica, permaneceram vivos, produzindo diásporas, bem como atraindo dispersores. Outro aspecto refere-se ao fato da área estar inserida em um grande contínuo florestal, o que contribui consideravelmente para a rápida recolonização do ambiente.

\section{Referências Bibliográficas}

Bortoluzzi, R.L.C. 2000. Papilionoideae (Leguminosae) no Parque Estadual do Rio Doce, Minas Gerais, Brasil. Dissertação de Mestrado. Universidade Federal de Viçosa, Viçosa, MG.

Brower, J.E. \& Zar, J.H. 1984. Field and laboratory methods for general ecology (Second edititon). Wm. C. Brown Company, Iowa. 226p.

Brumitt, R.K. \& Powell, C.E. 1992. Authors of plant names. Royal Botanic Gardens - Kew, Whitstable Litho Ltd.: Whitstable, Kent, Great Britain. 732p.

Cavassan, O. 1983. Levantamento fitossociológico da vegetação arbórea da mata da Reserva Estadual de Bauru, utilizando o método de quadrantes. Bauru, Sp: Faculdades do Sagrado Coração (Cadernos de Divulgação Cultural, 4). 81p.

CETEC. 1978. Levantamento pedológico do Parque Estadual do Rio Doce. Fundação Centro Tecnológico de Minas Gerais - CETEC: Belo Horizonte, MG. 68p.

CETEC. 1982. Levantamento da vegetação do Parque Estadual do Rio Doce. Fundação Centro Tecnológico de Minas Gerais - CETEC: Belo Horizonte, MG. (não paginado).

Cottam, G. \& Curtis, J.T. 1956. The use of distance measures phytosociological sampling. Ecology 37:451-460.

Cronquist, A. 1981. An integrated system of classification of flowering plants. New York: Columbia University. 1.262p.

Feio, R.N., Braga, Ú.M.L., Wiederhecker, H., Santos, P.S. 1998. Anfíbios do Parque Estadual do Rio Doce (Minas Gerais). Universidade Federal de Viçosa, Instituto Estadual de Florestas. 32p.: il.

Felfili, J.M. 1993. Structure and dynamics of a gallery forest in Central Brazil. Tese de Doutorado. Oxford University, Oxford.

Gilhuis, J.P. 1986. Vegetation survey of the Parque
Florestal Estadual do Rio Doce, MG, Brasil. Dissertação de Mestrado. Universidade Federal de Viçosa, Viçosa, MG.

Gonçalves, A.P.S. 2000. Bambus (Bambusoideae s.l.: Poaceae) no Parque Estadual do Rio Doce - MG, Brasil. Dissertação de Mestrado. Universidade Federal de Viçosa, Viçosa, MG.

Graçano, D., Prado, J., Azevedo, A.A. 1998. Levantamento preliminar de Pteridophyta do Parque Estadual do Rio Doce (Minas Gerais). Acta Botanica Brasilica 12(2):165-181.

IEF. 1994. Pesquisas prioritárias para o Parque Estadual do Rio Doce, Brasil. Belo Horizonte, MG: Instituto Estadual de Florestas - IEF. 35p.

Lawrence, G.H.M. 1951. Taxonomia das Plantas Vasculares. Lisboa: Fundação Calouste Gulbenkian, v.2, 854p. (Tradução da Ed. Macmillan Co, 1951)

Leitão Filho, H.F. (Coord.). 1993. Ecologia da Mata Atlântica em Cubatão (SP). São Paulo, SP: Editora da Universidade Estadual Paulista; Campinas: Editora da Universidade Estadual de Campinas. 184p.

Martins, F.R. 1991. Estrutura de uma floresta mesófila. Campinas, SP: Universidade Estadual de Campinas. 241p.

Meira-Neto, J.A.A., Martins, F.R. 2000. Estrutura da Mata da Silvicultura, uma Floresta Estacional Semidecidual Montana no município de Viçosa, MG. Revista Árvore 24(2):151-160.

Melo, M.M.R.F., Mantovani, W. 1994. Composição florística e estrutura de trecho de mata atlântica de encosta, na ilha do Cardoso (Cananéia, SP, Brasil). São Paulo: Instituto de Botânica (Boletim do Instituto de Botânica, 9). 158p.

Meyer, H.A., Recknagel, A.B., Stevenson, D.D., Bartoo, R.A. 1961. Forest management. 2.ed. New York: Ronald. 282p.

Mota, L.P. 1995. Distribuição diamétrica fitossociológica - Diamfito. Universidade Federal de Viçosa, Viçosa, MG. 2p.

Mueller-Dombois, D., Ellenberg, H. 1974. Aims and methods of vegetation ecology. New York: John Wiley \& Sons. 547p.

Pagano, S.N., Leitão Filho, H.F., Shepherd, G.J. 1987. Estudo fitossociológico em Mata Mesófila Semidecídua, no município de Rio Claro (Estado de São Paulo). Revista Brasileira de Botânica 10(1):49-62.

Pedralli, G., Teixeira, M.C.B., França, E.G. 1986. Lauraceae do Parque Florestal do Rio Doce, Minas Gerais. Ciência e Cultura 38:1.414-1.421. 
Peixoto, A.L., Rosa, M.M.T., Joels, L.C.M. 1995. Diagramas de perfil e de cobertura de um trecho da Floresta de Tabuleiro na Reserva Florestal de Linhares (Espírito Santo, Brasil). Acta Botanica Brasilica 9(2): 177-194.

Pielou, E.C. 1975. Ecological diversity. New York: John Wiley \& Sons. 165p.

Royal Botanic Gardens - Kew. 1993. Index Kewensis on compact disc - manual. Oxford University Press, Oxford.

Scudeller, V.V., Carvalho-Okano, R.M. 1998. Bignonieae (Bignoniaceae) no Parque Estadual do Rio Doce, Minas Gerais, Brasil. IHERINGIA, Sér.Bot., 51(1):79-133.

Shepherd, G.J. 1994. Fitopac 1 - manual do usuário. Campinas, SP: Universidade Estadual de Campinas. 88p.

Silva, A.F., Fontes, N.R.L., Leitão Filho, H.F. 2000. Composição florística e estrutura horizontal do estrato arbóreo de um trecho da Mata da Biologia da Universidade Federal de Viçosa - Zona da Mata de Minas Gerais. Revista Árvore 24(4):397-405.

Silva, A.F., Leitão Filho, H.F. 1982. Composição florística e estrutura de um trecho de mata atlântica de encosta no município de Ubatuba (São Paulo, Brasil).
Revista Brasileira de Botânica 5(1/2):43-52.

Silva Júnior, M.C., Silva, A.F. 1988. Distribuição dos diâmetros dos troncos das espécies mais importantes do cerrado na Estação Florestal de Experimentação de Paraopeba (EFLEX) - MG. Acta Botanica Brasilica 2(1-2):107-126.

SOCT \& CETEC. 1981. Programa de pesquisas ecológicas no Parque Estadual do Rio Doce. Sistema Operacional de Ciência e Tecnologia - SOCT \& Fundação Centro Tecnológico de Minas Gerais CETEC: Belo Horizonte, MG. v.2. (Relatório final)

Souza, A.L., Jesus, R.M. 1994. Distribuição diamétrica de espécies arbóreas da Floresta Atlântica: análise de agrupamento. Viçosa, MG: Sociedade de Investigações Florestais (Boletim Técnico, 10). 30p. il.

Spiegel, M.R. 1977. Estatística. São Paulo: McGrawHill do Brasil. 580p.

Temponi, L.G. 2001. Estudo taxonômico e distribuição das Araceae do Parque Estadual do Rio Doce, Minas Gerais, Brasil. Dissertação de Mestrado. Universidade Federal de Viçosa, Viçosa, MG.

Veloso, H.P., Rangel Filho, A.L.R., Lima, J.C.A. 1991. Classificação da vegetação brasileira, adaptada a um sistema universal. Rio de Janeiro: IBGE. 124p. 